\title{
SDRT and Continuation Semantics
}

\author{
Nicholas Asher ${ }^{1}$ and Sylvain Pogodalla ${ }^{2}$ \\ 1 IRIT, CNRS \\ nicholas.asher@irit.fr \\ 2 LORIA/INRIA Nancy - Grand Est \\ sylvain.pogodalla@inria.fr
}

\begin{abstract}
Segmented Discourse Representation Theory (SDRT) $[2,7]$ provides a dynamic semantics for discourse that exploits a rich notion of discourse structure. According to SDRT, a text is segmented into constituents related to each other by means of rhetorical relations; the resulting structure, known as a segmented discourse representation structure or SDRS has various semantic effects. This theory has shown how discourse structure makes contributions to the interpretation of a variety of linguistic phenomena, including tense, modality, presupposition, the interpretation of anaphoric pronouns and ellipsis. SDRT exploits dynamic semantics $[20,14]$ to interpret SDRSs. We investigate here the advantages of integrating SDRT within continuation style semantics of the sort developed in [17].
\end{abstract}

Keywords: SDRT, dynamic semantics, continuation semantics

\section{An introduction to continuation semantics}

Most versions of dynamic semantics (DS) in linguistics make heavy use of assignment functions as semantic objects. In this SDRT is not different. While this may not be apparent to the casual reader of early work like [20], a compositional semantics for DRT, DPL [14] or more recent developments [11] leads almost inevitably to the introduction of "odd" types whose inhabitants are variables, assignments or other "representational" elements. Dynamic Intensional Logic (DIL) [15, 12], following [19]'s work on programming languages, has the virtue of making central the semantic status of assignment functions, as they are the points of evaluation in that model theory. But almost all of extant versions of DS include assignment functions as parts of semantic values. The exploitation of assignment functions as semantic objects plays a crucial role in dynamic semantic approaches to discourse interpretation. This engenders subtle differences in the underlying logic, clouding the logical status of discourse referents in a "top" DRS by making them appear ambiguous between existentially bound variables and free ones (cf. [13]). It also leads to problems of destructive assignment in DPL and with variable clash in DRT or versions of DPL that use partial assignment functions. It is difficult to avoid these problems in a purely compositional environment, leading to cumbersome systems. 
Continuation style semantics (CS) developed by computer scientists in the 70 s and introduced into linguistics by $[9,26,17,10]$ avoid these problems. CS provides a more abstract setting for dynamic logics, abstracting away from assignments that are essential to the formulations of DIL, DPL and DRT. CS, like DS, models the dynamic meaning of a natural language expression as a transition between a left context and a right one. But in CS right contexts are explicitly introduced and are defined in terms of left contexts and sentence denotations. CS exploits the structure of a monad in category theory [23], which specifies the parameters needed to provide a CS: the first is to specify what a left context is; the second is to provide a "binder" rule, which tells us how to combine the semantics of a text with that of subsequent sentences; the third is to specify the lexical entries for expressions. CS thus refocuses semanticists' attention on specifying appropriate lexical entries and discourse contexts. CS permits a wide variety of choices as to what left contexts, binder rules and lexical entries are, a liberalism we exploit below.

As an example, [17]'s CS exploits Montague's homomorphic interpretation of syntactic types and structures into semantic types and terms. But [17] changes Montague's interpretation of the sentence type $s, \llbracket s \rrbracket$, from $t$ to $\Omega \triangleq \gamma \rightarrow(\gamma \rightarrow$ $t) \rightarrow t$, where $\gamma$ is the type of the left context or discourse context already given and $\gamma \rightarrow t$ is the type of the right context or discourse "to come" its continuation. If this sentence introduces a new discourse entity $x$, given an environment $i$ (as input) and a continuation $k$ as parameter, it can provide $(x:: i)$ (with · : $\cdot$ a list constructor of type $e \rightarrow \gamma \rightarrow \gamma$ ) as parameter to $k$, making the value of $x$ available for $k$.

Other types have standard interpretations. Where $\llbracket X \rrbracket$ stands for the $\lambda$-term or meaning of $X, \llbracket n p \rrbracket=(e \rightarrow \llbracket s \rrbracket) \rightarrow \llbracket s \rrbracket$ and $\llbracket n \rrbracket=e \rightarrow \llbracket s \rrbracket$. Pronouns have the following interpretation: $\llbracket i t \rrbracket=\lambda P . \lambda i k . P(\operatorname{sel} i) i k$, where $i: \gamma, k: \gamma \rightarrow t$, and where $\operatorname{sel} i$ is a function that selects a suitable discourse antecedent inside $i$.

A CS like [17]'s must say how a text $T$ combines with a sentence to its right. This is the CS binder issue, which is also an essential part of all dynamical systems - it is the DRS update operation of DRT or relational composition of sentence contents in DPL. Here is the basic binder equation for de Groote's system and ours:

$$
\llbracket T . S \rrbracket=\lambda i . \lambda k \cdot \llbracket T \rrbracket i\left(\lambda i^{\prime} \cdot \llbracket S \rrbracket i^{\prime} k\right)
$$

That is, the text to date $T$ takes the meaning of $S$ as its right context, or rather the meaning of $S$ suitably applied and abstracted so that it can be of $t$ type. A quick type check on $\lambda i^{\prime} \cdot \llbracket S \rrbracket i^{\prime} k$ confirms that this is indeed the right output: $\llbracket S \rrbracket: \gamma \rightarrow(\gamma \rightarrow t) \rightarrow t$ so $\lambda i^{\prime} . \llbracket S \rrbracket i^{\prime} k: \gamma \rightarrow t$.

Let's look at a sample discourse to see how the theory works.

(2) A man is sleeping. He is snoring.

Suitable lexical entries provide a completely classical interpretation of these two sentences except that they have both a left and right context and that the second sentence fills in the right context of the first. The entries also make the existential determiner introduce an individual into the right context - a witness that will 
be "selected" by the pronoun. With this in mind, the interpretation of the first sentence is:

$$
\lambda i . \lambda k . \exists x .(\operatorname{man} x) \wedge(\text { sleeping } x) \wedge(k(x:: i))
$$

The second sentence provides the $\lambda$-term:

$$
\lambda i . \lambda k \text {. (snoring }(\operatorname{sel} i)) \wedge(k i)
$$

Using the binder rule in (1), these last two $\lambda$-terms yield a meaning for (2) :

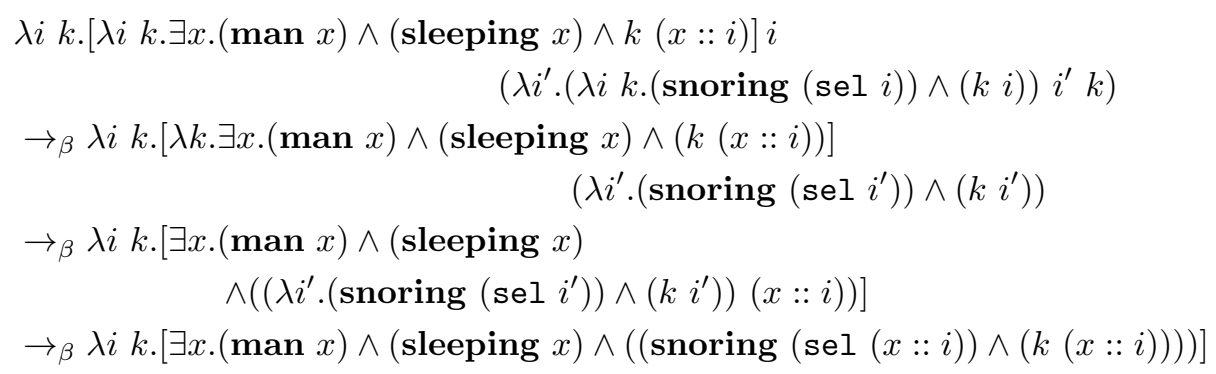

In selecting the bound variable $x$, the semantic value of the anaphoric pronoun falls within the scope of the original existential quantifier, reducing anaphoric binding to quantificational binding.

In [8], we show how de Groote's proposal differs from Dynamic Intensional Logic and Dynamic Montague Grammar, which appear to be close cousins. Nevertheless, there are the fundamental differences we have noted above between the two approaches. We argue in this paper that CS in the style of $[17,24,8]$ can profit SDRT in several ways, by generalizing the theory, reducing a dependence on descriptions of representations and integrating syntax and discourse in an interesting way.

\section{An Introduction to SDRT in Continuation Semantics}

SDRT, as we have said, is a theory that exploits a rich notion of discourse structure. But what is this? For SDRT, as for most theories that investigate discourse structure, such structure involves units or discourse constituents that are linked by discourse relations that define the rhetorical function of the constituent in the discourse. Let us look at some examples.

(5) a. John walked in. • He poured himself a cup of coffee.

b. John fell. • Mary pushed him.

c. We bought the apartment, $\bullet$ but we've rented it.

d. Il commence à dessiner et peindre en $1943, \bullet$ fréquente les ateliers de sculpture $\bullet$ puis de peinture de l' école des Beaux-Arts d' Oran, $\bullet$ où il rencontre Guermaz (ANNODIS corpus). 
e. Julie had an excellent meal, • beginning with an elegant and inventive truffes du Périgord en première cuisson comme un petit déjeuner, $\bullet$ followed by some wonderful scallops, $\bullet$ then sweetbreads, $\bullet$ a sumptuous cheese plate, $\bullet$ and ending with a scrumptious dessert.

A presumption of relevance leads us to infer some link between elementary discourse units or EDUs (clauses or subclausal units whose boundaries are marked by $\bullet$ in the examples above). These links involve relations that are familiar even to the non-linguist: some units elaborate or go into more detail concerning something introduced in another constituents (these are Elaboration type relations) as in (5e); some units form a parallel or a contrast with other units (such units are linked by Parallel or Contrast), as in (5c); some units furnish explanations why something described in another unit happened (Explanation) as in (5b); and some units constitute a narrative sequence of events (Narration) (5a) or (5d).

Some discourse relations are encoded grammatically through the use of certain grammatical constructions (like adverbial or purposive clauses, parentheticals or left fronted temporal or spatial adverbials $)^{3}$ or through discourse connectors like as a result, puis or the choice and sequencing of lexical items. An example of a set of discourse relations triggered by the choice of verb and complement comes in (5e), with the use of beginning with, followed by and ending with. Sometimes, it is less clear what linguistic source triggers the inference of the discourse relation as in (5a-b) - most likely, an as yet not fully understood mix of lexical semantics and world knowledge. The discourse relations implicated by these devices have impose structural constraints on the discourse context and have truth conditional effects that a number of researchers have explored. ${ }^{4}$

To construct a discourse structure for a text, we must accomplish three tasks:

- to segment a text into EDUs;

- to compute attachment points of EDUs in a discourse structure;

- to compute one or more discourse relations between an EDU and its attachment point(s).

An SDRT discourse structure or SDRS is the result of these computations and may contain complex constituents where several EDUs combine together to make one larger constituent. An SDRS is a logical form for discourse with a well-defined dynamic semantics that has many equivalent formulations - as a first order model like structure consisting of a set of labels and assignments of formulas to labels [7], as a DRS like structure [2] or as we will show below, a $\lambda$-term in intensional logic.

To get an idea of what SDRSs look like consider the following text (6) discussed at length in [7]. The model-like SDRS is given in (7).

\footnotetext{
${ }^{3}$ For a discussion of these, see for instance [27].

${ }^{4}$ With regards to temporal structure, see [22]; on pronominal anaphora, see [2,21]; on presupposition see [6]; on sluicing and ellipsis see $[2,5,25]$.
} 
(6) $\quad \pi_{1}$. John had a great evening last night.

$\pi_{2}$. He had a great meal.

$\pi_{3}$. He ate salmon.

$\pi_{4}$. He devoured lots of cheese.

$\pi_{5}$. He then won a dancing competition.

(7) $\langle A, \mathcal{F}$, Last $\rangle$ where: $\left\{\begin{array}{l}A=\left\{\pi_{0}, \pi_{1}, \pi_{2}, \pi_{3}, \pi_{4}, \pi_{5}, \pi_{6}, \pi_{7}\right\} \\ \mathcal{F}\left(\pi_{0}\right)=\text { Elaboration }\left(\pi_{1}, \pi_{6}\right) \\ \mathcal{F}\left(\pi_{6}\right)=\operatorname{Narration}\left(\pi_{2}, \pi_{5}\right) \wedge \text { Elaboration }\left(\pi_{2}, \pi_{7}\right) \\ \mathcal{F}\left(\pi_{7}\right)=\operatorname{Narration}\left(\pi_{3}, \pi_{4}\right) \\ \text { Last }=\pi_{5}\end{array}\right.$

In SDRT we can abstract away from the details of the structure to get a graph representation, which is relevant to computing discourse accessibility (again for details see [7]):

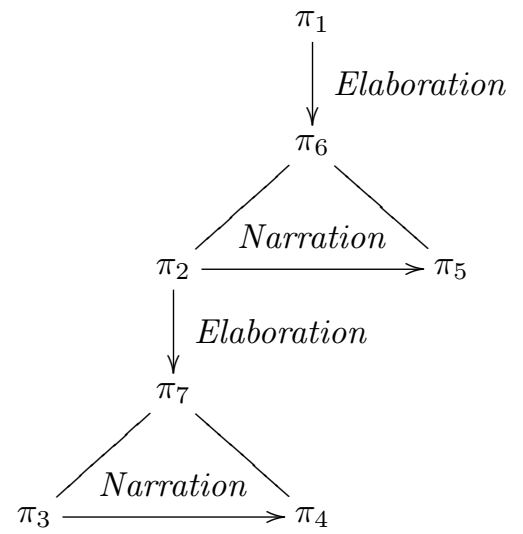

Notice that some discourse relations are represented as vertical lines in the graph whereas others are horizontal arcs; these correspond to two different types of relations - subordinating and coordinating relations, and these two types of relations affect anaphoric and attachment possibilities differently. ${ }^{5}$

Inferring discourse relations to build an SDRS is a matter of defeasible and uncertain inference. Many of the features used to infer discourse relations are only good indications of a particular discourse relation or particular discourse structure; very few are in and of themselves sufficient to deductively infer the relation or structure. Many discourse connectives are for example ambiguous. In addition, many segments may bear discourse relations to other segments despite the lack of discourse connectives or known structural or lexical cues, as in (5a,b) or (6). SDRT uses a nonmonotonic logic, a logic for defeasible inference, tailored to inferring discourse relations. We will appeal to this as an oracle in what follows below.

\footnotetext{
${ }^{5}$ For a discussion, see for example $[3,7]$.
} 
We now turn to a more logic based representation of discourse structures. As proposed in [4] and then further developed in [7], SDRSs can be represented using a labelled language, where the labels stand for discourse constituents. In such a language every $n$-ary predicate becomes an $(n+1)$-ary predicate.

In keeping with earlier work, we assume the language is that of IL together with a set of labels $\pi, \pi_{1}, \pi_{2}, \ldots$ of atomic type $\ell$, representing discourse constituents, and a set of relation symbols of type $\ell \rightarrow \ell \rightarrow \ell \rightarrow t$ that represent discourse relations over constituents. We write $R\left(\pi_{1}, \pi_{2}, \pi\right): t$ to state that the discourse relation $R$ holds between $\pi_{1}$ and $\pi_{2}$ in constituent $\pi$. These formulas will be introduced during combination process. This process for reasons developed in [7] makes appeal to a separate reasoning module known as the Glue Logic.

An SDRS in CS is a formula or a lambda term of type $\Omega$, the dynamic type of sentences and texts in [17] and [8]. In this framework, we need to specify:

- the nature of $\gamma$;

- the binder rule for combining a text and a new constituent;

- and a lexicon.

We define the type $\gamma$ as records as in [8], the fields of which are:

- a set of Labels;

- a set of Accessible Labels;

- a set of accessible Discourse Entities;

- and a proposition of type $t$.

The latter, which is redundant with the overall result, is needed to be part of the context so that we can make inferences based on the content of the context. ${ }^{6}$ SDRT makes the accessibility of a discourse entity dependent upon the discourse structure; we capture this by making the accessible discourse entities for the information to come dependent on the label of attachment - thus our field for discourse entities will be pairs of accessible labels with other variables (for discourse entities). We provide several functions on records that retrieve needed information.

$-\operatorname{sel}_{L}: \gamma \rightarrow \ell$ extracts a label from the left context that is SDRT accessible (for a definition of SDRT accessibility see e.g., [7]). ${ }^{7}$

$-\operatorname{sel}_{E}: \gamma \rightarrow \ell \rightarrow e$ extracts a discourse referent from the set of accessible discourse referents associated with a label. ${ }^{7}$

$-\operatorname{sel}_{\rho}: \gamma \rightarrow \ell \rightarrow \ell \rightarrow \ell \rightarrow t$. This function is used to pick a discourse relation (i.e. a ternary relation) linking a label chosen from $i$, the current context, and returns a proposition.

${ }^{6}$ We might rather consider a hyperintensional type PROP to make these inferences, but we don't want to focus on that point here. Such inferences may be required in example such as $(5 \mathrm{~b})$ to get the right temporal order and the right discourse relation which may depend upon the context.

${ }^{7}$ Using the techniques introduced in [24], we could express this function using only an update operator on sets of labels/entities. 
$-v: \gamma \rightarrow \ell \rightarrow \gamma$. This is the update function that changes the left context record in virtue of the information contained in $S$ and the linking of its label to some label in $i$ via a chosen discourse relation. This update function is defined in terms of SDRT's glue logic which operates on fields of a left context. We write the update operation as $v\left(i, \pi_{S}\right)$, where $S$ is the current discourse constituent and $i$ is a left context.

We now present the operation of combining a discourse with a sentence. Several new operations are involved at this level: first an application of $\mathrm{sel}_{L}$, then an application of $\operatorname{sel}_{\rho}$, and a use of the update operation $v$. Note that in order to clearly show how bound variables, both for segment labels and discourse referents, can be added to the context during the process, we explicitly use the function :: in the first next examples rather than the $v$ function that somehow "hide" the underlying modifications of the context. This :: function is an overloaded function with type either $\ell \rightarrow \gamma \rightarrow \gamma$ or $e \rightarrow \gamma \rightarrow \gamma$. Our binder rule for SDRT is just that of $[17]^{8}$ which is:

$$
\llbracket D . S \rrbracket=\lambda i o \pi \cdot \exists \pi_{1} \cdot \llbracket D \rrbracket\left(\pi_{1}:: i\right)\left(\lambda i^{\prime} \cdot \exists \pi_{2} \cdot \llbracket S \rrbracket\left(\pi_{2}:: i^{\prime}\right) o \pi_{2}\right) \pi_{1}
$$

However, the semantics of a sentence is more complex, since it must attach to the text meaning with one or more discourse relations. Which relations are involved will depend on the structure of the discourse to date and the left context. The discourse relations define a partial order over labels. We say that if $R\left(\pi_{1}, \pi_{2}, \pi_{3}\right)$, then $\pi_{3}>\pi_{1}$ and $\pi_{2}>\pi_{1}$. A constraint of well-foundedness says that $>$ always has a unique maximal element and that $>$ is asymmetric and transitive; this ensures that we never have $R\left(\pi_{1}, \pi_{2}, \pi_{1}\right)$ or $R\left(\pi_{1}, \pi_{2}, \pi_{2}\right)$. The $v$ function should take this into account.

The standard interpretation for a sentence $S$ expressing some predicate $P_{S}$ in [17] is: $\llbracket S \rrbracket=\lambda i o \pi \cdot P_{S} \wedge\left(o i^{\prime} \pi\right)$, that is, in addition to providing the content $P_{S}$ to the discourse, it transforms $i$ into $i^{\prime}$ (e.g. adding some new discourse referent with :: or $v$ ) and gives its to its continuation. Because discourse relations now come into play, the interpretation we get is in general rather:

$$
\llbracket S \rrbracket=\lambda i o \pi \cdot \exists \pi_{S} \cdot P_{S} \wedge \operatorname{sel}_{\rho}\left(\operatorname{sel}_{L}(i), \pi_{S}, \operatorname{sel}_{L}(i)\right) \wedge\left(o i^{\prime} \pi\right)
$$

where $i^{\prime}=v\left(i, \pi_{2}\right)$ consists in $i$ plus the new discourse label and the new discourse relation (plus possible new discourse referents, of course), provided the constraints on $>$ are met. The kind of reasoning done in [7] will tell us how to update $i$ via the Glue Logic and get $i^{\prime}$.

There are however some exceptions to this schema. Indeed the rule (9) sometimes results in an inconsistency because the discourse context updated by $D$ has only one label in it (one element in Labels), or even none in the case of the first sentence. In this case, we cannot form a formula $R\left(\pi_{i}, \pi_{S}, \pi_{j}\right)$ that is

\footnotetext{
${ }^{8}$ With the slight modification that now $\Omega \triangleq \gamma \rightarrow(\gamma \rightarrow \ell \rightarrow t) \rightarrow \ell \rightarrow t$ so that we can anchor a segment using a segment label.
} 
consistent with the constraints on $>$. In these cases, the interpretation also introduces an auxiliary label ${ }^{9}$ and we treat this exception to (9) with the following interpretation:

$$
\llbracket S \rrbracket=\lambda i o \pi \cdot \exists \pi^{\prime} \cdot \exists \pi_{S} \cdot P_{S} \wedge \operatorname{sel}_{\rho}\left(\operatorname{sel}_{L}(i), \pi_{S}, \pi^{\prime}\right) \wedge\left(o i^{\prime} \pi\right)
$$

The case where there is no label at all in the context (starting sentence) is dealt with the same way, except that its semantic representation is limited to the predicate (there is no additional discourse relation).

By adding further constraints about discourse structure, for example those involving complex segments, the nature of discourse relations or topics, (9) may generate other exceptions that we may specify analogously.

\subsection{Lexicalized Discourse Relation}

Let's illustrate a simple case with a lexicalized discourse relation. We compute the semantic representation of (11) using the lexicon of Table 1. In this lexicon, we integrate the fact that theses sentences occur at the first and the second position directly in the lexicon.

(11) $\left(\pi_{1}\right)$ A man walked in. $\left(\pi_{2}\right)$ Then he coughed.

Table 1. A simple lexicon

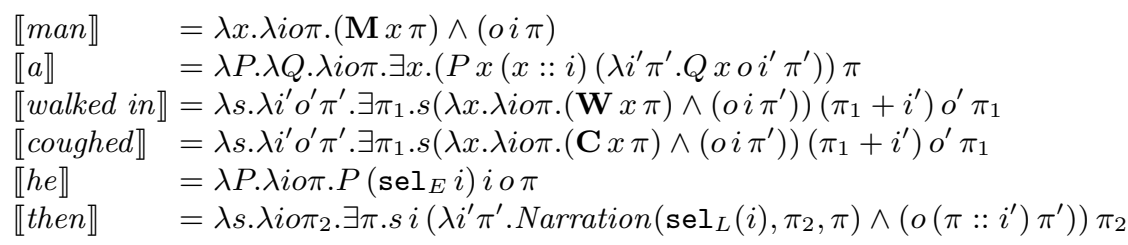

\footnotetext{
${ }^{9}$ For sake of clarity we assume here another kind of interpretation. However, the kind of exception mechanism of [18] for presupposition could be used. Here, the presupposition that needs to be accommodated is the existence of a suitable label $\pi$.
} 
We can then compute the following interpretations:

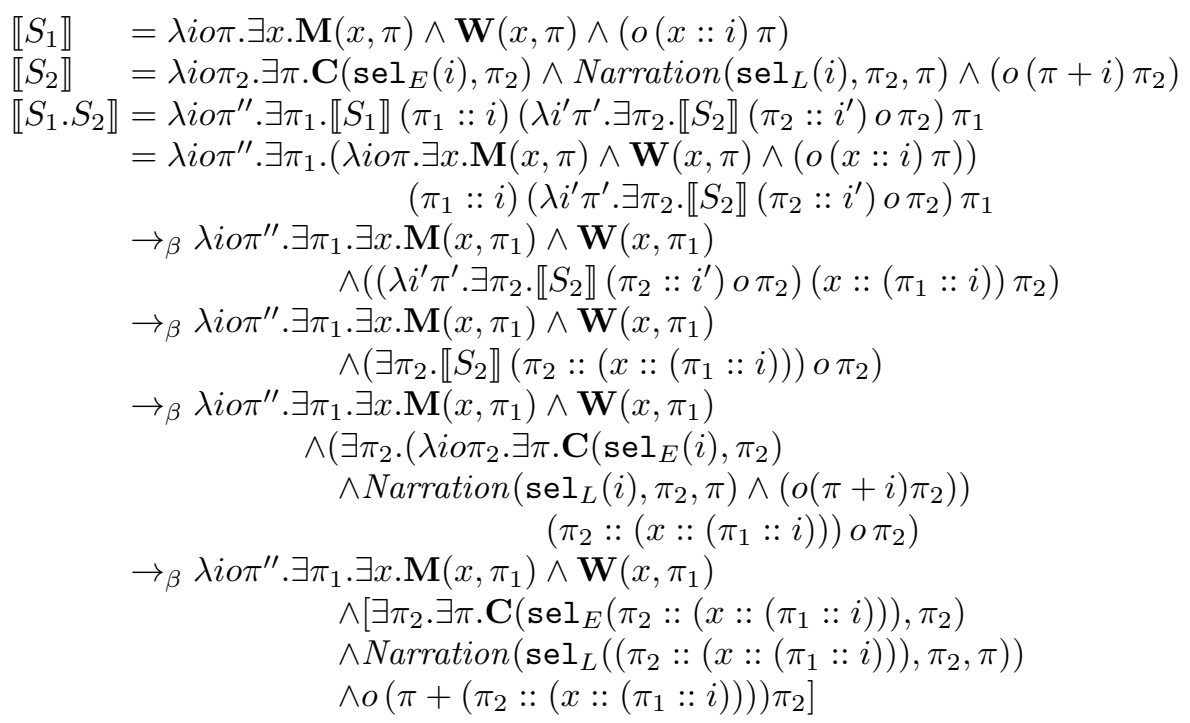

\subsection{Getting More General}

In this section, we don't develop the computation steps anymore. We want to stress here how we can structure the context and how it evolves. Example (12) is very similar to (11) except that there is no lexicalization of the discourse relation. (12) $\left(\pi_{1}\right)$ A man walked in. $\left(\pi_{2}\right)$ He coughed.

Eventually, the discourse relation between $\pi_{1}$ and $\pi_{2}$ is set to Narration (meaning $\operatorname{sel}_{\rho}\left(\operatorname{sel}_{L}\left(i^{\prime}\right), \pi_{2}, \pi\right)$ gets resolved in $\left.\operatorname{Narration}\left(\pi_{1}, \pi_{2}, \pi\right)\right)$.

The stages in the computation reveal the evolution of the left context as the discourse is processed. Supposing that we have a record $i_{0}$ with empty fields for contents, discourse entities and discourse labels, the first sentence provides us with an update to the left context as follows:

$$
\left[\begin{array}{ll}
\text { Labels }= & \left\{\pi_{1}\right\} \\
\text { Available Labels }= & \left\{\pi_{1}\right\} \\
\text { Discourse entities }= & \left\{\left(\pi_{1},\{x\}\right)\right\} \\
\text { Content }= & \exists \pi_{1} \cdot \exists x \cdot \mathbf{M}\left(x, \pi_{1}\right) \wedge \mathbf{W}\left(x, \pi_{1}\right)
\end{array}\right]
$$

After the update with the second sentence of (12), we assume that

$$
\operatorname{sel}_{E}\left(v\left(v\left(i_{0}, \pi_{1}\right), \pi_{2}\right), \pi_{2}\right)=x
$$

and the context is now:

$$
\left[\begin{array}{ll}
\text { Labels }= & \left\{\pi_{1}, \pi_{2}, \pi\right\} \\
\text { Available Labels }= & \left\{\pi_{2}, \pi\right\} \\
\text { Discourse entities }= & \left\{\left(\pi_{1},\{x\}\right),\left(\pi_{2},\{x\}\right)\right\} \\
\text { Content }= & \exists \pi_{1} \cdot \exists x \cdot\left(\mathbf{M}\left(w, \pi_{1}\right) \wedge \mathbf{W}\left(x, \pi_{1}\right)\right) \wedge \exists \pi \cdot \exists \pi_{2} . \mathbf{C}\left(x, \pi_{2}\right) \\
& \wedge \text { Narration }\left(\pi_{1}, \pi_{2}, \pi\right)
\end{array}\right]
$$


(14) provides for a suitable antecedent for the pronoun he because the selection function $\mathrm{sel}_{E}$ can find the appropriate antecedent in the list of discourse entities of the current record. Note that the set of available nodes calculated for a given left context exploits the definition in [7]. Here because Narration is a coordinating relation, it makes $\pi_{1}$ not an available label, following [24]. In contrast, the discourse (15a) provides a different picture. Let's suppose that $\pi_{2}$ attaches to $\pi_{1}$ with Background, which is a subordinating discourse relation. Intuitively, we would like to leave open both for the possibility of continuing the elaboration or description of the man or by talking about something that is linked to the first constituent, as in $(15 \mathrm{~b}, \mathrm{c})$. To allow for all these attachments the update of an empty discourse context with (15a) yields the left context in (16) for future continuations.

(15) a. $\left(\pi_{1}\right)$ A man walked in. $\left(\pi_{2}\right)$ It was raining.

b. $\left(\pi_{1}\right)$ A man walked in. $\left(\pi_{2}\right)$ It was raining. $\left(\pi_{3}\right)$ He wanted to buy a new suit.

c. $\left(\pi_{1}\right)$ A man walked in. $\left(\pi_{2}\right)$ He sported a hat. $\left(\pi_{3}\right)$ Then a woman walked in. $\left(\pi_{4}\right)$ She wore a coat.

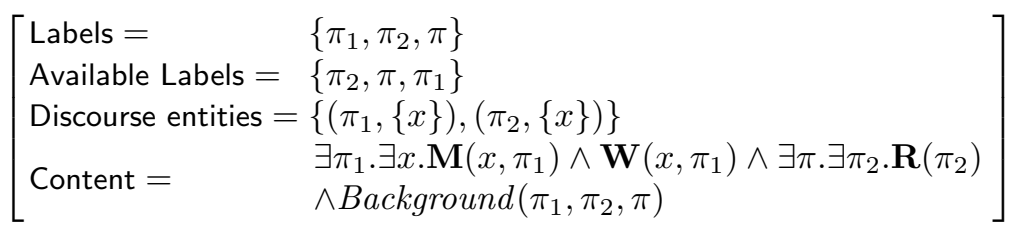

On the other hand, (15c) yields the left context in (17):

$$
\left[\begin{array}{rl}
\text { Labels }= & \left\{\pi_{1}, \pi_{2}, \pi, \pi_{3}, \pi^{\prime}, \pi_{4}\right\} \\
\text { Available Labels }= & \left\{\pi^{\prime}, \pi_{3}, \pi_{4}\right\} \\
\text { Discourse entities }= & \left\{\left(\pi_{4},\{y, c\}\right),\left(\pi_{3},\{y\}\right)\right\} \\
& \exists \pi_{1} \cdot \exists x \cdot \mathbf{M}\left(x, \pi_{1}\right) \wedge \mathbf{W}\left(x, \pi_{1}\right) \wedge \\
& \exists \pi \cdot \exists \pi_{2} \cdot \exists h \cdot \mathbf{S}\left(\operatorname{sel}_{E}\left(x:: \operatorname{nil}, \pi_{1}\right), h, \pi_{2}\right) \wedge \mathbf{H}(h) \\
& \wedge \text { Background }\left(\pi_{1}, \pi_{2}, \pi\right) \wedge \\
& \exists \pi_{3} \cdot \exists y\left(\mathbf{W o}\left(y, \pi_{3}\right) \wedge \mathbf{W}\left(y, \pi_{3}\right)\right) \\
& \wedge \exists \pi^{\prime} . \operatorname{Narration}\left(\pi, \pi_{3}, \pi^{\prime}\right) \wedge \\
& \exists \pi_{4} \cdot \exists c \cdot \text { Wear }\left(\operatorname{sel} l_{E}\left(y:: \operatorname{nil}, \pi_{3}\right), c, \pi_{4}\right) \\
& \wedge \text { Coat }\left(c, \pi_{4}\right) \wedge \text { Background }\left(\pi_{3}, \pi_{4}, \pi^{\prime}\right)
\end{array}\right]
$$

Notice that given the attachments in $(15 \mathrm{c})$, the man $x$ and its hat $h$ are no longer an accessible discourse entities for future continuations, even though the existential quantifier introduced in $\pi_{1}$ has scope over the content introduced by $\pi_{4}$ and possible continuations. Our reformulation of SDRT in CS thus makes the right frontier constraint of SDRT, cf. $[2,7,1]$, follow from the semantics, which was not the case in earlier work. A left context specifies an SDRS graph and other more familiar representations of SDRSs used in the literature. Nevertheless, the record formalism is more general and flexible than other specifications of SDRT, and allows the addition of other fields, should they be needed in specifying discourse structure (for example questions under discussion). Further refinements 
of (8) will allow for multiple attachment points and links with multiple discourse relations, which SDRT allows.

\section{A Refinement of the Binder Rule for SDRT}

Our continuation style semantics for SDRT allows us to specify more concretely the semantics of discourse relations. For example, consider the distinction made in [7] between veridical and non veridical discourse relations. [7] argues that one cannot have $R(a, b, l) \wedge R^{\prime}(b, c, l)$, where $R$ is veridical and $R^{\prime}$ is not veridical but its treatment of the labelled language as a description language did not permit it to really say why such a formula is problematic. For us, this formula is contradictory. We have assigned labels the atomic type $\ell$; but in a richer typed framework, we can be more specific: we can take them to be nominalizations of propositions that can be localized - hence some sort of facts or possibilities. We hold that certain discourse relations can affect the type of the labels they relate. We have already made clear that there is a function from labels to their contents (we'll call it $\|$.$\| ), as discourse related constituents produce an ordinary formula$ in the labelled language, a formula which has a standard model theoretic content. It is now straightforward to define (left and right) veridical and non veridical discourse relations:

$-R$ is veridical iff $R(a, b, l) \rightarrow\|l\| \subseteq\|a\| \wedge\|l\| \subseteq\|b\|$

- $R$ is non-right and left veridical iff $R(a, b, l) \rightarrow\|l\| \nsubseteq\|a\| \wedge\|l\| \nsubseteq\|b\|$

It is now evident that with a non-left and right veridical relation like Alternation, the following discourse structure is simply inconsistent:

$$
\text { Contrast }(a, b, l) \wedge \text { Alternation }(b, c, l)
$$

We can use the TEST operation of [8] and attempt to use the binder rule in the way above. If the result is consistent, the binder rule can be used as above. However, if the evaluation of the application of the rule yields an inconsistent structure, then we may specify an EXCEPTION condition that explicitly introduces a new constituent for a discourse like

$$
\left(\pi_{1}\right) \text { John likes sports but }\left(\pi_{2}\right) \text { Bill doesn't. }\left(\pi_{3}\right) \text { Or Sam doesn't }
$$

so that we get the discourse structure in $(20)$ :

$$
\operatorname{Contrast}\left(\pi_{1}, \pi, l\right) \wedge \operatorname{Alternation}\left(\pi_{2}, \pi_{3}, \pi\right)
$$

which is intuitively what is desired.

\section{From Syntax to Discourse}

Another important reason for exploiting the CS framework is to get a tighter connection between sentential syntax and discourse semantics, something that [7] 
simply does not treat. The continuation style semantics used in $[17,24,8]$ are all tightly coupled to syntactic theories elaborated in the Abstract Categorial Grammar (ACG) framework [16]. We believe that it will be fruitful to use ACGs to study how syntactic structures can affect discourse semantics, as well as clausal semantics. For example, elements on the left periphery of an IP, parentheticals, can be specified in the syntax semantics interface to generate discourse constituents. Other syntactic and lexical constructions appear useful to analyze in the CS framework:

(21) a. In the thirties, liquor could not be sold in most areas. Speakeasies developed throughout the US [detached IP adverbial with scope over the subsequent sentence]

b. Stock shares fell today, partly because of investor anxiety about the weak recovery.

c. John came to the party, only because he couldn't think of anything else to do.

d. He betrayed and then murdered your father [Obi-Wan Kenobe to Luke Skywalker]

e. I disagree with the honorable Senator's motion for three fundamental reasons. . .

All of these examples offer interesting examples of the interplay between syntax, lexical semantics and discourse semantics. Frame adverbials studied in [27] like in the thirties and enumeration structures like that announced in (21e) introduce a novel element via lexical and syntactic constructions: they introduce a relation that holds between the label for the material in the adverbial or the clause in (21e) and a label in the discourse to come. Thus, our selection functions must select from future continuations, something that is relatively straightforward in continuation semantics but that requires a heavy-handed use of underspecification in standard formulations of SDRT. Among the various account to handle this case, we could add a new field to the context that, in addition to the content $\left(\exists \pi_{1}\right.$.in_the_thirties $\left.\left(\pi_{1}\right)\right)$, could contain (a list of) pending relations, introduced by the left adjoined adverbial and that would need to be emptied under some conditions by the next segments, such as:

$$
\lambda \pi . \lambda i . E l a b o r a t i o n\left(\pi_{1}, \pi, \operatorname{sel}_{L}(i)\right) \wedge o(i)
$$

In (21b-d), we have examples where syntactic structure incorporates lexical elements like because or and then that introduce discourse structure. We believe that a framework like CS can treat these cleanly as well.

\section{References}

1. Afantenos, S., Asher, N.: Testing SDRT's right frontier. In: 23rd International Conference on Computational Linguistics (COLING 2010) (2010)

2. Asher, N.: Reference to Abstract Objects in Discourse. No. 50 in Studies in Linguistics and Philosophy, Kluwer, Dordrecht (1993) 
3. Asher, N.: Troubles on the right frontier. In: Khnlein, P., Benz, A. (eds.) Constraints in Discourse (CID 2005). Pragmatics \& Beyond New Series, vol. 172, pp. 29-52. John Benjamins Publishing Company (2008)

4. Asher, N., Fernando, T.: Effective labeling for disambiguation. In: Bunt, H. (ed.) Second International Workshop in Computational Linguistics. Tilburg, The Netherlands (1997)

5. Asher, N., Hardt, D., Busquets, J.: Discourse parallelism, ellipsis, and ambiguity. Journal of Semantics 18(1), 1-25 (2001)

6. Asher, N., Lascarides, A.: The semantics and pragmatics of presupposition. Journal of Semantics 15(3), 239-299 (1998)

7. Asher, N., Lascarides, A.: Logics of Conversation. Cambridge University Press (2003)

8. Asher, N., Pogodalla, S.: A montagovian treatment of modal subordination. In: Semantics and Linguistic Theory 20 (SALT 20) (2010)

9. Barker, C.: Continuations in natural language. In: 4th Continuations Workshop (2004)

10. Bernardi, R., Moortgat, M.: Continuation semantics for the Lambek-Grishin calculus. Information and Computation 208(5), 397-416 (2010), Special Issue: 14th Workshop on Logic, Language, Information and Computation (WoLLIC 2007)

11. Brasoeavnu, A.: Structured anaphora to quantifier domains. Information and Computation (to appear)

12. Dekker, P.: Scopes in discourse. Journal of Language and Computation 1, 7-32 (1999)

13. Fernando, T.: What is a DRS? Tech. Rep. R2.1.B, Dyana deliverable (1994), also in the proceedings of a workshop on Computational Semantics (Tilburg, The Netherlands), December 1994

14. Groenendijk, J., Stokhof, M.: Dynamic predicate logic. Linguistics and Philosophy 14(1), 39-100 (1991)

15. Groenendijk, J., Stokhof, M.: Changing the context: Dynamics and discourse. In: et al, E.D. (ed.) 11th Annual Conference and of the Workshop on Discourse (IATL 3). The Israel Association for Theoretical Linguistics, Jerusalem (1996)

16. de Groote, P.: Towards Abstract Categorial Grammars. In: Association for Computational Linguistics (ACL), 39th Annual Meeting and 10th Conference of the European Chapter (EACL). pp. 148-155 (2001)

17. de Groote, P.: Towards a Montagovian account of dynamics. In: Semantics and Linguistic Theory 16 (SALT 16) (2006)

18. de Groote, P., Lebedeva, E.: Presupposition accomodation as exception handling. In: 11th Annual SIGdial Meeting on Discourse and Dialogue (SIGDIAL 2010) (2010)

19. Janssen, T.: Foundations and Applications of Montague Grammar. Ph.D. thesis, University of Amsterdam (1983)

20. Kamp, H.: A theory of truth and semantic representation. In: Groenendijk, J.A., Janssen, T., Stokhof, M. (eds.) Formal Methods in the Study of Language. Foris (1981)

21. Kehler, A., Kerta, L., Rohde, H., Elman, J.: Coherence and coreference revisited. Journal of Semantics 25(1), 1-44 (2008), special Issue on Processing Meaning

22. Lascarides, A., Asher, N.: Temporal interpretation, discourse relations and commonsense entailment. Linguistics and Philosophy 16(5), 437-493 (1993)

23. Moggi, E.: Notions of computation and monads. Information and Computation 93(1), 55-92 (1991) 
24. Pogodalla, S.: Exploring a type-theoretic approach to accessibility constraint modelling. In: Journées Sémantique et Modélisation. Toulouse (2008)

25. Romero, M., Hardt, D.: Ellipsis and the structure of discourse. Journal of Semantics 21(4), 375-414 (2004)

26. Shan, C., Barker, C.: Explaining crossover and superiority as left-to-right evaluation. Linguistics and Philosophy 29(1), 91-134 (2006)

27. Vieu, L., Bras, M., Asher, N., Aurnague, M.: Locating adverbials in discourse. Journal of French Language Studies 15(2), 173-193 (2005) 